\title{
Circuit based anti-correlation, attention orienting, and major depression
}

\author{
Philippe Fossati*
}

Institut du Cerveau et de la Moelle Epinière, ICM, Paris, France; Inserm, U 1127, Paris, France; CNRS, UMR 7225, Paris, France; Sorbonne Université, Paris France; AP-HP, Hôpital de la Pitié Salpêtrière, Service de Psychiatrie d'Adultes, Paris, France

\begin{abstract}
Major depression is a multidimensional disorder producing emotional dysregulation, cognitive impairment, and neurovegetative symptoms. A pathophysiological model of depression needs to explain how these dimensions interact to produce specific clinical phenotypes and how these interactions may predict remission to specific treatments. It is unlikely that major depression results from discrete brain lesions. Here we propose to define major depression as a disorder of neural networks. We review evidence suggesting that the dynamics of neural networks involved in allocation of attention resources to the internal and external world contribute to cognitive impairment, increased self-focus, and dysfunctional saliency detection in depression. We describe cognitive and emotional tasks that reveal abnormal cooperation between the Central Executive Network and the Default Mode Network. Finally we suggest that depression is associated with increased social rejection sensitivity. Studies on social rejection will shed light on how attachment relates to impairment in allocation of attention resources to produce depressive symptoms such as rumination and cognitive problems.
\end{abstract}

Received 1 February 2018; Accepted 24 September 2018; First published online 30 January 2019

Key words: Attention orienting, brain network, default mode network, saliency, social rejection.

\section{Introduction}

Major depression is a highly prevalent and costly disorder. ${ }^{1}$ Although several treatments are available, only $40 \%$ of depressed patients receiving antidepressant or psychotherapy achieve remission with initial treatment $^{2}$ and almost $30 \%$ of depressed patients fail to respond to several trials of antidepressant drugs. Among others, one main reason put forward to explain the resistance to antidepressant treatment is the absence of well-defined pathophysiology of major depression.

A major depressive episode assimilates symptoms from different domains or dimensions, such as emotional symptoms (sadness, anhedonia), cognitive symptoms (attention problems, executive impairment, rumination), and neuro-vegetative symptoms (anorexia and sleep disorders). A good pathophysiological model of major depression needs to explain not only these different symptoms but also how they interact to produce a specific clinical phenotype.

Major depression is now considered to be a brain disorder. In a classical perspective, the term brain

* Address for correspondence: Philippe Fossati, Department of Adult Psychiatry, GH Pitié Salpétrière 47-83 bd de l'Hopital Paris 75013 Cedex. (Email: philippe.fossati@aphp.fr) disorder normally refers to medical conditions associated with discrete lesions. This neurological model is unlikely to reflect the complexity, the diversity, and the specificity of depressive clinical phenotypes.

Therefore it has now been proposed that major depression is associated with dysfunctions of large-scale functional and structural brain networks. Networks are defined here by inter-temporal and regional relationships between brain regions during resting state and tasks. The development of brain imaging techniques and data analyses has helped us to define very well the brain architecture and neural networks that govern perceptive, cognitive, and emotional processes. Likewise several models of dysfunctional brain networks have been proposed in major depression. ${ }^{3,4}$ It is beyond the scope of this paper to review in details these models.

Here we analyzed the literature on the functional connectivity and dynamics of neural networks involved in allocation of attention resources to the internal and external world in relation with major depression. Our general idea is that major depression and risk for depression are associated with difficulties in adequately allocating brain resources, in term of orientation and quantity, toward the internal and/or external world in response to specific cognitive and emotional demands. 
This difficulty in responding to specific demands reveals impairment in the cooperation and synchronization of specific brain networks, namely the default mode network and the central executive network that precipitate and reinforce depressive symptoms such as emotional bias, rumination, and cognitive problems. We will describe emotional and cognitive tasks that may reveal this abnormal networks dynamic. Finally we discuss the role of one stressful situation, social exclusion, and threat to social acceptance in the dynamic of these brain networks and the brain signature of major depression.

\section{Allocation of Attention Resources and Brain Networks}

Our attention could be directed externally toward watching a soccer game on TV and at a certain moment suddenly be directed internally to memories or to plan for the coming holiday. This distinction between externally directed attention and internally directed attention has increasingly focused research interest in the cognitive literature.

Dixon et $a l^{5}$ in a recent review suggested that these two types of attention orienting may be in a trade-off competition or may co-occur at the same time depending on the level of intentional or involuntary processing and cognitive resources engaged. Moreover they emphasized that both modes of attention processing have segregated and common neural signatures. The externally directed attention is related to the central executive network (CEN), which includes the lateral prefrontal cortex, parietal cortex, and insula. The CEN supports working memory and attention directed to the external world, whereas the default mode network (DMN) supports internal mental activity and attention directed toward the internal world. The DMN includes the posterior cingulate cortex (PCG), precuneus, inferior parietal cortex (IPG), and medial prefrontal cortex (mPFC).

We have elsewhere largely demonstrated the role of the DMN, with a special emphasis on its anterior part (mPFC), in internally oriented attention, especially selfreferential processing that relates neutral or emotional stimuli to the self.

Memory tasks can be used to electively study these self-related processes. In one task we can ask subjects to judge whether positive or negative personality traits described them or not (ie, the "self" condition), or whether it described a generally desirable trait or not (ie, the "general" condition). Usually words processed in the self-condition are better recalled or recognized than words encoded in a semantic mode, an effect known as the self-reference effect (SRE) in the literature on memory. ${ }^{6}$

Combining this task with fMRI, we first demonstrated that the medial prefrontal cortex (dorsal part) was more activated in the self-condition compared to the general condition, regardless of the emotional valence of the words. ${ }^{7}$ Several studies replicated these findings emphasizing the importance of medial prefrontal cortex and anterior part of the DMN in internally oriented attention. $^{8}$ Likewise autobiographical memory processes engaged during retrieval of personal events activated the anterior and posterior parts of the DMN, with a specific role for precuneus in perspective taking. ${ }^{9,10}$

Both attention modes, internally oriented and externally oriented, recruit the lateral prefrontal cortex (mainly in its dorsal part) during intentional processing and may enter into a competition for cognitive resources, meaning that at a high level of cognitive demands priority needs to be defined in allocation of resources. Below I hypothesize that depressed patients and subjects at risk for depression have difficulties with prioritizing the attention investment in order to cope with cognitive and emotional demands.

\section{Cooperation Within and Between Brain Networks and Cognitive Tasks}

We have learned from functional brain imaging data that it is difficult to relate specific brain regions to specific cognitive functions, given the dependence of cerebral activity on the anatomical connections between distant brain regions. This is the classical distinction between functional segregation on one side and functional integration on the other side. ${ }^{11}$ From a functional integrative perspective, cognitive and emotional processes result from the cooperation within and between brain networks and not only from local brain regions.

One particular cognitive task, the N-Back, illustrates well this perspective. During the N-Back task, subjects are required to match a stimulus (a letter or a number) to stimuli previously presented 1 (1-back), 2 (2-back), or 3 (3-back) trials before. Subjects need to constantly monitor and to update the content of their working memory, and usually the performance monotonically decreases from 1-back to 3 -back, reflecting the increase of cognitive demands. We used this task in healthy controls with neutral material (letter) and reward feedback during fMRI acquisition. ${ }^{12}$ As expected, subjects showed bilateral activations in the CEN: DLPFC (BA 9/46), premotor and SMA (BA 6/8), Broca area, dorsal anterior cingulate cortex (ACC), and parietal cortex. Activations of these regions were modulated by the complexity of the task (from 1- to 3-back condition). Increasing cognitive demand engages a pattern of brain activation characterized by a balance between increasing activity in cortical cognitive areas (CEN) and decreasing activity in the limbic and paralimbic structures such as ventro-medial prefrontal regions $\left(\mathrm{DMN}^{12}\right)$. 
How can we explain the deactivation of DMN during the N-Back task? Two general hypotheses may put be forward. According to the first, in order to satisfy the cognitive demand, the subject needs to inhibit personal thoughts (emotional or neutral) that may interfere with the performance to the task. This "content hypothesis" suggests that the subject disengages self-related processes during the N-Back task, as reflected by DMN deactivation. With the second hypothesis, consistent with Dixon et $a l$ 's view on allocation of attention resources, ${ }^{5}$ the cognitive demand of the task increases the competition for cognitive resources in a brain system with limited capacity. Consequently brain activity is amplified in networks representing relevant information (here the CEN) and is suppressed in networks representing irrelevant information (here the DMN). According to this context hypothesis, the DMN deactivation during the N-Back task is independent of thought content.

Both hypotheses suggest that CEN and DMN act in concert and/or in opposition, as they have been shown to be anti-correlated during both cognitive tasks and during resting state. ${ }^{13}$ Moreover the quality of cooperation between the DMN and the CEN may explain intrasubject variability in working memory performance in healthy subjects. $^{14}$

The content and context hypotheses require us to define the saliency of external and internal stimuli and to prioritize the orientation of attention resource investment. The salience network (SN), which includes the ventro-lateral prefrontal cortex, anterior insula, and the dorsal anterior cingulate cortex, supports this integration of external and internal salient stimuli to guide behaviors and defines priority. The studies of temporal dynamics between different networks have shown that the fronto-insular cortex from the $\mathrm{SN}$ is critical for switching from CEN to DMN and vice versa. ${ }^{15}$ In this triple network model, interaction and synchronization of $\mathrm{DMN}, \mathrm{SN}$, and CEN are major determinants in saliency detection, orientation of attention resources, and performance variability during effortful cognitive task. ${ }^{16}$

\section{Brain Networks Dynamics, Cognitive Problems, and Major Depression}

Two biases characterize cognitive problems in depression: the effortful cognitive bias and the increased selffocus bias.

The effortful cognitive bias reflects the difficulty of depressed patients to perform cognitive tasks that require cognitive resources (ie, tasks with cognitive processes accomplished in sequence and restricted by the short-term memory capacity) leaving intact automatic processes in several domains such as learning, memory, problem-solving, reading, and speed processing (for a review, see Hartlage $e t a l^{17}$ ).

The effortful-deficit bias in depression predicts impairment in the actions requiring attention and executive resources, such as complex goal-directed behaviors. We suggest that deficits of depressed patients on effortful tasks are preceded by increasing effort to maintain a high level of performance. The progressive exhaustion of cognitive resources precedes the deficits of depressed patients, and the reduction of cognitive resources is a final by-product of the failure of depressed patients to constantly adapt to cognitive demands. fMRI data support that hypothesis.

In a fMRI study with a verbal N-Back task, ${ }^{18}$ we compared 10 depressed subjects and 10 normal controls. We elsewhere showed that depressed patients have decreased performance level on the 3 conditions of the N-Back task compared to healthy controls. ${ }^{19}$ In our fMRI study, we a priori selected depressed patients with normal performance on the N-Back task and no difference between groups was found for both performance and reaction times for each levels of complexity of the task. Both groups, depressed and controls, showed bilateral activations of the CEN. Activations of these regions were modulated by the complexity of the task. Within this N-Back neural network, depressed patients showed greater activation of the DLPFC and dorsal anterior cingulate than normal controls. Since this seminal work, several fMRI studies used an N-Back task or a working memory task in depressed patients. Some of these studies replicated our original findings and showed a hyperactivation in the left DLPFC in depressed patients as compared to normal controls. ${ }^{20-22}$

The aberrant activation of the DLPFC and anterior cingulate associated with normal performance in depressed subjects may indicate different problems: (1) inefficiency of a task-related neural network with difficulty to organize neural activity and abnormal signal-to-noise ratio due to dopaminergic or glutamatergic dysfunction, ie, the "context" hypothesis; (2) structural brain abnormalities within the working memory network; (3) excess of subjective effort (volition) or subject's task engagement; (4) difficulties in inhibiting activation in limbic structures and personal concerns during the cognitive task as postulated with the content hypothesis; and (5) greater needs to monitor the putative errors and conflict than controls reflected by a greater activation of the ACC.

We looked at the deactivation in the DMN in our N-Back study. Depressed patients have more difficulties that normal controls to deactivate the medial prefrontal cortex activity during the cognitive effort. ${ }^{18} \mathrm{~N}$-Back level modulated the DMN deactivation: the higher the cognitive demand the greater the deactivation. 
Impairment in working memory task is a cognitive marker of acute depression and may persist in remitted depressed patients. ${ }^{22}$ Some recent data showed that an impaired neural signature of working memory task may also be present in subjects at risk for depression before the occurrence of any depressive episode. ${ }^{23}$

In a recent study, ${ }^{24}$ we assessed more precisely the temporal dynamics between the CEN and DMN during the N-Back task in remitted depression in relationship with residual emotional symptoms.

More specifically we assessed within and between connectivity of the CEN and DMN in remitted depressed patients with and without emotional blunting while performing the N-back task. In order to control for treatment effect, all remitted patients were treated with the SSRI paroxetine. We hypothesized that emotional blunting in remitted depressed patients would be associated with abnormal cooperation between the DMN and CEN, hence producing greater intrasubject variability of performance on the N-back task.

Consistent with this hypothesis, the dynamics of cooperation between the CEN and the DMN differed between the two remitted depressed groups. Compared to the other group, the patients with residual depressive symptoms showed a significant decreased anticorrelation between DMN and the CEN during the N-back task performance (see Figure 1). Moreover, this decreased anti-correlation was associated with increased performance variability in the N-Back task.

Increased self-focus is a second bias in major depression. We have emphasized in several publications that increased self-focus in depression is related to abnormal activity within the DMN with a special role for the medial prefrontal cortex. $^{25,26}$ The self-focus bias associated with negative emotional experience enhances the integration of negative stimuli to the self and contributes to the classical self-blame and decreased self-esteem of depressed patients. Rumination, a repetitive thinking on the causes and consequences of emotion associated with self-referential processing, is the hallmark of this increased self-focus bias. ${ }^{27}$

Whereas increased self-focus is associated with increased connectivity within the $\mathrm{DMN}$, rumination involves increased connectivity of the sub-genual part of the anterior cingulated cortex to both the anterior and posterior parts of the DMN. ${ }^{28}$

In the previous study with the N-Back task, ${ }^{24}$ we introduced in the analyses of the cooperation between DMN and CEN, the ruminative scores of remitted depressed patients assessed with ruminative rating scales. In emotionally blunted depressed patients with residual symptoms, the higher the anti-correlation (negative correlation) between the DMN and the CEN, the lower the ruminative scores. Overall in remitted

Functional connectivity during $\mathrm{n}$-back task

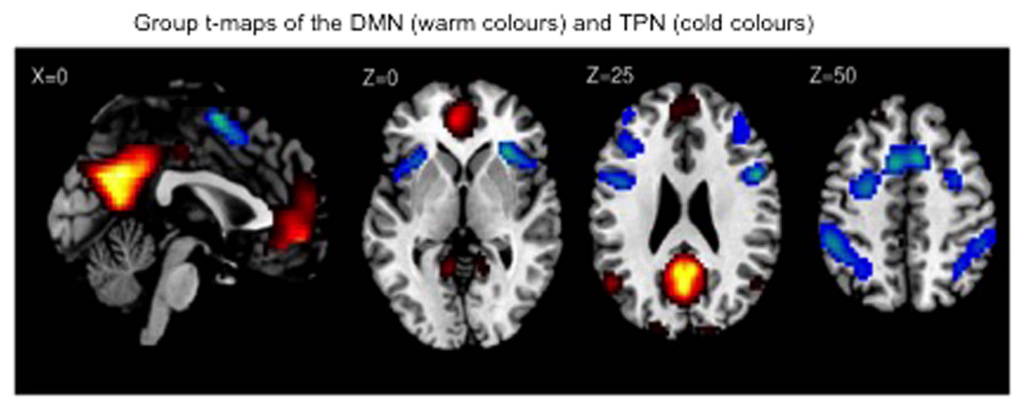

Networks of interest
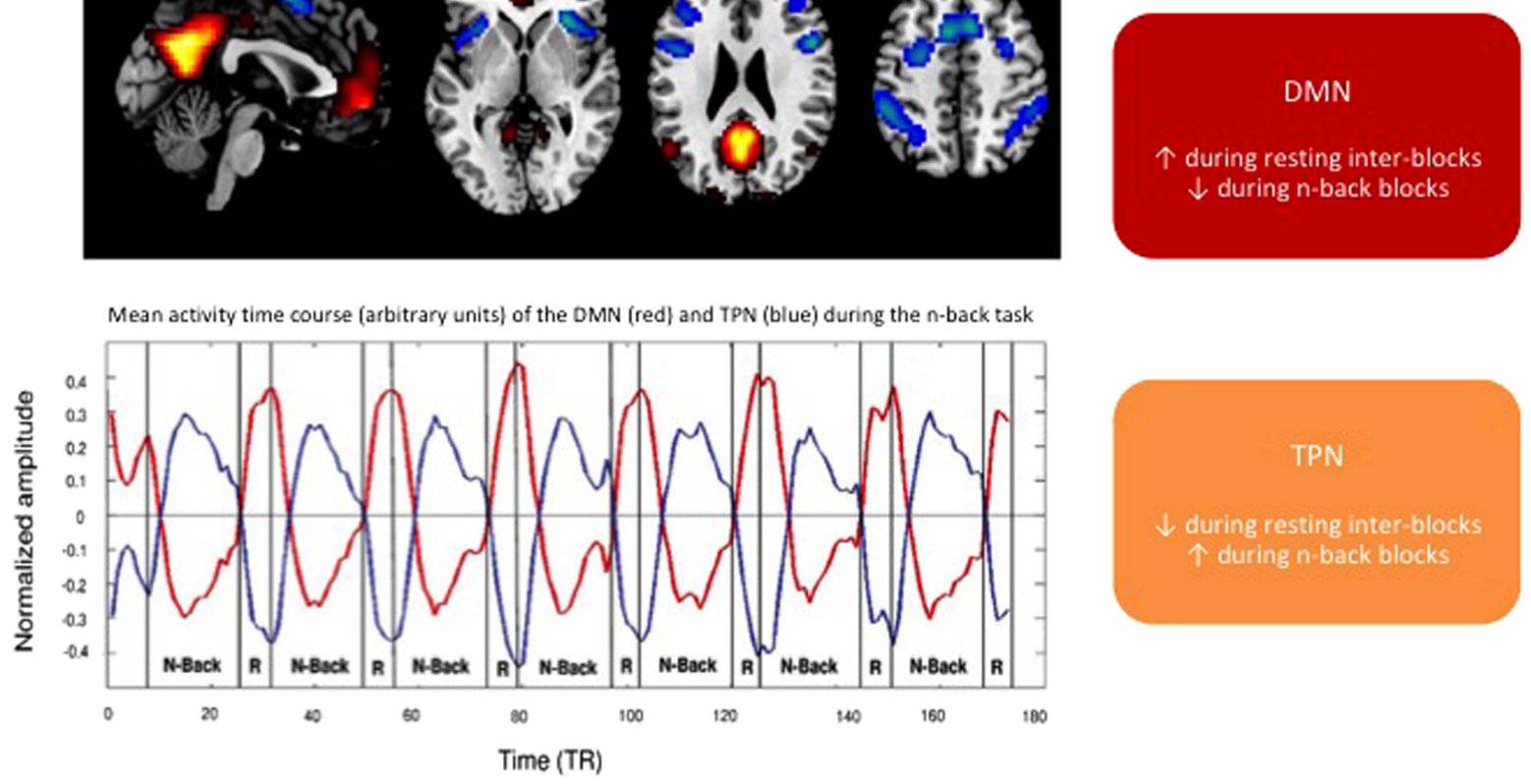

FIGURE 1. Anticorrelation of DMN and TPN networks during the N-Back task in remitted depressed patients with or without residual emotional symptoms (adapted from Delaveau et al.). ${ }^{24}$ DMN: default mode network; TPN: task positive network; TR: repetition time. 
depressed patients with residual emotional symptoms, the cooperation between CEN and DMN is involved in coping with ruminative thinking rather than monitoring their performance.

We described that during both cognitive and emotional tasks, the cooperation between the CEN and DMN is impaired in depression, leading to difficulties in adequately allocating cognitive resources, variability of performance in effortful cognitive tasks, and abnormal self-processing as revealed by rumination. This abnormal network dynamic persists during remission and represents core features of depression.

Beyond brain regions that constitute the DMN and CEN, the sub-genual anterior cingulate cortex (SgACC) is a key player in depression and in treatment of depression ${ }^{29,30}$. As proposed by Hamilton et al, ${ }^{28}$ the increased connectivity between the SgACC and the DMN underlies rumination in depression.

Below we propose new pathophysiological pathways that may explain on one side the abnormal cooperation between DMN andCEN and on the other side the increased connectivity between SgCCA and DMN in depression.

\section{Rejection Sensitivity, Rumination, and Depression}

Interpersonal factors are among the strongest predictors of the onset and course of a major depressive episode. However little attention has been paid to the biological mechanisms of interpersonal difficulties of depressed patients.

Social exclusion - being kept apart from others - and Threat to Social Acceptance - threat resulting from social rejection, exclusion or ostracism - are interpersonal stressors that may precipitate or reinforce a depressive episode.

To explore emotional, cognitive, behavioral, and biological reactions to social rejection, the Cyberball game became a gold standard in the last several years. ${ }^{31}$ Cyberball is a minimal social interactive ball game in which participants are ignored and excluded by 2 other players. During the game, the participant is led to believe that he plays a ball-tossing game with 2 other real players through the internet. In fact, the reactions of these 2 other players are computer-generated to induce feelings of exclusion in the participant. In the exclusion condition, the participant will generally get the ball no more than 2 or 3 tosses, whereas in the inclusion condition, the participant will get $30 \%$ of the tosses.

Many brain imaging studies using this Cyberball paradigm showed that social rejection triggers a brain response in the ventro-lateral prefrontal cortex, anterior cingulate cortex, and anterior insula, which are regions that usually process the negative affect and the distress associated with physical pain. ${ }^{32}$ A meta-analysis by our group recently demonstrated that the ventral part of the anterior cingulate, the subgenual cingulate (SgACC), is particularly associated with self-reported distress triggered by social rejection. ${ }^{33}$

In order to cope with the negative emotional consequences of social exclusion, individuals need to mobilize cognitive resources and self-regulatory strategies. After social exclusion, subjects may prioritize the allocation of attention resources toward the "external world" and toward the processing of social cues that signal opportunity for social connection (affiliative behaviors) or for social withdrawal (defensive behaviors).

After social exclusion, subjects may also allocate attention resources toward the internal world and increase self-focus attention. Thus, as a self-protection strategy, people downplay their negative personality traits and exaggerate their positive traits when facing threat to social acceptance. ${ }^{34}$

Self-evaluative processes and increased self-focused attention triggered by social exclusion may also be maladaptive by reinforcing and maintaining the negative affect resulting from this social stress. The subject sometimes activates rumination after exclusion.

Relationships between sensitivity to social rejection and rumination are complex and bidirectional. Rejection sensitivity is a dispositional trait characterized by anxious expectation of being rejected, readily perception of signal of rejection, and overreaction to rejection. ${ }^{35}$

Both rejection sensitivity (assessed by the Rejection Sensitivity Questionnaire) and rumination are risk factors for depression. Rumination is associated with increased interpersonal difficulties (social withdrawal, submissive interpersonal relationships), and subjects frequently ruminate on interpersonal problems.

A prospective study by Pearson et $a l^{36}$ examined the longitudinal relationships between rumination and rejection sensitivity in currently depressed, previously depressed, and never depressed subjects. Baseline rejection sensitivity prospectively predicted increased rumination 6 months later after controlling for baseline rumination and depression. Alternatively, baseline rumination did not predict rejection sensitivity. Overall the results suggest that rumination may represent a consequence rather than a cause for rejection sensitivity.

People with high levels of rejection sensitivity are at risk for depression, and depressed patients have high levels of rejection sensitivity. Two specific features characterized people with high rejection sensitivity: (1) subjects expect to be rejected and (2) subjects put a high value on preventing social rejection. According to this formulation, the "high expectancy-high value" susceptibility determines specific situations that can precipitate a depressive episode: situations where subjects cannot 


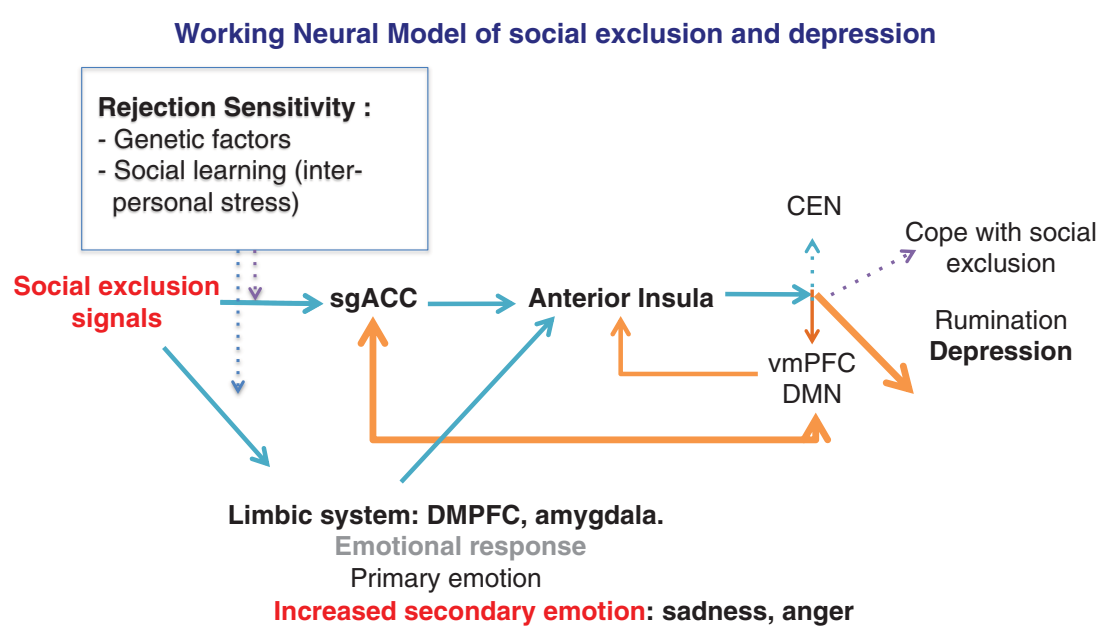

FIGURE 2. General model showing how rejection sensitivity, exposure to social stressors, and allocation of attentional resources may precipitate a depressive episode. Orange arrows indicate a depressive response after stress exposure. Blue arrows indicate normal responses to social stress. SgACC: subgenual anterior cingulate; VMPFC: ventromedial prefrontal cortex; DMPFC: dorsomedial prefrontal cortex; DMN: default mode network; CEN: central executive network.

control rejection. Epidemiological studies have illustrated that the break of an important relationship increases significantly the risk for depression, especially if this break is not initiated by the subject. ${ }^{37}$ Consistent with this, in a 6-month longitudinal study of college-age women high in rejection sensitivity compared to those who are low, those high in rejection sensitivity became more depressed when they experienced a partnerinitiated breakup but not when they experienced a selfinitiated or mutually initiated breakup. ${ }^{38}$

Following unique or repeated exposures to threat to social acceptance, subjects high in rejection sensitivity produce several emotional responses, including sadness and anger. At the cognitive level, subjects high in rejection sensitivity will more likely activate ruminative processes and increase their self-focused attention. Rumination in this context reflects both the failed goal of preventing the social rejection perceived as a personal failure and the response to the secondary emotion (sadness and/or anger) associated with the loss induced by social rejection.

At the neural level, we speculate that social exclusion will activate the SgACC as a detector of social rejection. The SgACC transfers the signal information to the anterior (and dorsal part) of the insula that calls for regulation. According to the triple network model, ${ }^{16}$ the anterior insula regulates the coordination between the DMN and the CEN. Instead of synchronizing the CEN-DMN dynamics following social exclusion, subjects at risk for depression or with a personal history of depression show increased connectivity between the DMN and the SgACC, as well as increased rumination. ${ }^{28}$ Rumination will then dampen the executive resources and reinforce the negative emotional response. This will result in difficulties in adequately allocating attentional resources toward the external world in order to cope with the social stress (see Figure 2).

Some of our propositions need to be validated and further explored. It has to be demonstrated that social exclusion abnormally involved the CEN-DMN cooperation in subjects with high rejection sensitivity. However, preliminary results in healthy volunteers confirm the adaptive changes of large scale brain networks after exposure to the Cyberball exclusion. ${ }^{39}$ Likewise the specific roles of the SgACC and anterior insula in detection and regulation, respectively, during processing of social exclusion signal need to be tested.

\section{Conclusion}

We have briefly reviewed the literature that views major depression as a disorder of the functional connectivity and dynamics of neural networks involved in allocation of attention resources to the internal and external world. Several situations detect the abnormal cooperation between DMN and CEN in depression, including the $\mathrm{N}$-Back task and the Cyberball game. We postulated that depression is not simply the result of selective regional or pathway dysfunctions, but also involves failure of the brain networks to maintain homeostatic cognitive control in times of increased cognitive demands. Because they require prioritizing one's attention orientation toward the internal or external (social) world, interpersonal stressors are exquisite factors that reveal abnormal network cooperation in depressed patients and in subjects at risk for depression. Moreover, the studies on the effects of these stressors shed light on how attachment relates to impairment in allocation of attention resources to produce depressive symptoms such as rumination and cognitive problems. 


\section{Disclosures}

Philippe Fossati has nothing to disclose.

\section{REFERENCES:}

1. Andlin Sobocki P, Wittchen HU. Cost of affective disorders in Europe. Eur J Neurol. 2005; 12(s1): 34-38. doi:10.1111/j.14681331.2005.01195.x.

2. Rush AJ, Trivedi MH, Wisniewski SR, et al. Bupropion-SR, sertraline, or venlafaxine-XR after failure of SSRIs for depression. $N$ Engl J Med. 2006; 354(12): 1231-1242. doi:10.1056/ NEJMoa052963.

3. Kaiser RH, Andrews-Hanna JR, Wager TD, Pizzagalli DA. Largescale network dysfunction in major depressive disorder. JAMA Psychiatry. 2015; 72(6): 603-611. doi:10.1001/ jamapsychiatry.2015.0071.

4. Williams LM. Precision psychiatry: a neural circuit taxonomy for depression and anxiety. Lancet Psychiatry. 2016; 3(5): 472-480. doi:10.1016/S2215-0366(15)00579-9.

5. Dixon ML, Fox KCR, Christoff K. A framework for understanding the relationship between externally and internally directed cognition. Neuropsychologia. 2014; 62: 321-330. doi:10.1016/j. neuropsychologia.2014.05.024.

6. Symons CS, Johnson BT. The self-reference effect in memory: a meta-analysis. Psychol Bull. 1997; 121(3): 371-394.

7. Fossati P, Hevenor SJ, Graham SJ, et al. In search of the emotional self: an fMRI study using positive and negative emotional words. $A m$ J Psychiatry. 2003; 160(11): 1938-1945. doi:10.1176/appi. ajp.160.11.1938.

8. Uddin LQ, Iacoboni M, Lange C, Keenan JP. The self and social cognition: the role of cortical midline structures and mirror neurons. Trends Cogn Sci. 2007; 11(4): 153-157. doi:10.1016/j. tics.2007.01.001.

9. Freton M, Lemogne C, Bergouignan L, Delaveau P, Lehéricy S, Fossati P. The eye of the self: precuneus volume and visual perspective during autobiographical memory retrieval. Brain Struct Funct. 2014; 219(3): 959-968. doi:10.1007/s00429-013-0546-2.

10. Fossati P. Imaging autobiographical memory. Dialogues Clin Neurosci. 2013; 15(4): 487-490.

11. Friston KJ. Functional and effective connectivity: a review. Brain Connect. 2011; 1(1): 13-36. doi:10.1089/brain.2011.0008.

12. Pochon JB, Levy R, Fossati $\mathrm{P}$, et al. The neural system that bridges reward and cognition in humans: an fMRI study. PNAS. 2002; 99(8): 5669-5674. doi:10.1073/pnas.082111099.

13. Damoiseaux JS, Rombouts SARB, Barkhof F, et al. Consistent resting-state networks across healthy subjects. Proc Natl Acad Sci U S A. 2006; 103(37): 13848-13853. doi:10.1073/pnas.0601417103.

14. Kelly AMC, Uddin LQ, Biswal BB, Castellanos FX, Milham MP. Competition between functional brain networks mediates behavioral variability. Neuroimage. 2008; 39(1): 527-537. doi:10.1016/j. neuroimage.2007.08.008.

15. Menon V, Uddin LQ. Saliency, switching, attention and control: a network model of insula function. Brain Struct Funct. 2010; 214 (5-6): 655-667. doi:10.1007/s00429-010-0262-0.

16. Menon V. Large-scale brain networks and psychopathology: a unifying triple network model. Trends Cogn Sci. 2011; 15(10): 483-506. doi:10.1016/j.tics.2011.08.003.

17. Hartlage S, Alloy LB, Vázquez C, Dykman B. Automatic and effortful processing in depression. Psychol Bull. 1993; 113(2): 247-278. doi:10.1037/0033-2909.113.2.247.

18. Harvey P-O, Fossati P, Pochon J-B, et al. Cognitive control and brain resources in major depression: an fMRI study using the n-back task.
Neuroimage. 2005; 26(3): 860-869. doi:10.1016/j. neuroimage.2005.02.048

19. Harvey PO, Le Bastard G, Pochon JB, et al. Executive functions and updating of the contents of working memory in unipolar depression. J Psychiatr Res. 2004; 38(6): 567-576. doi:10.1016/j. jpsychires.2004.03.003.

20. Rose EJ, Simonotto E, Ebmeier KP. Limbic over-activity in depression during preserved performance on the n-back task. Neuroimage. 2006; 29(1): 203-215. doi:10.1016/j. neuroimage.2005.07.002.

21. Matsuo K, Glahn DC, Peluso MAM, et al. Prefrontal hyperactivation during working memory task in untreated individuals with major depressive disorder. Mol Psychiatry. 2007; 12(2): 158-166. doi:10.1038/sj.mp.4001894.

22. Müller VI, Cieslik EC, Serbanescu I, Laird AR, Fox PT, Eickhoff SB Altered brain activity in unipolar depression revisited: meta-analyses of neuroimaging studies. JAMA Psychiatry. 2017; 74(1): 47-55. doi:10.1001/jamapsychiatry.2016.2783.

23. Mannie ZN, Harmer CJ, Cowen PJ, Norbury R. A functional magnetic resonance imaging study of verbal working memory in young people at increased familial risk of depression. Biol Psychiatry. 2010; 67(5): 471-477. doi:10.1016/j. biopsych.2009.10.006.

24. Delaveau P, Arruda Sanchez T, Steffen R, et al. Default mode and task-positive networks connectivity during the N-Back task in remitted depressed patients with or without emotional residual symptoms. Hum Brain Mapp. 2017; 38(7): 3491-3501. doi:10.1002/hbm.23603.

25. Lemogne C, le Bastard G, Mayberg H, et al. In search of the depressive self: extended medial prefrontal network during selfreferential processing in major depression. Soc Cogn Affect Neurosci. 2009; 4(3): 305-312. doi:10.1093/scan/nsp008.

26. Lemogne C, Delaveau P, Freton M, Guionnet S, Fossati P. Medial prefrontal cortex and the self in major depression. J Affect Disord. 2012; 136(1-2): e1-e11. doi:10.1016/j.jad.2010.11.034.

27. Nejad AB, Fossati P, Lemogne C. Self-referential processing, rumination, and cortical midline structures in major depression. Front Hum Neurosci. 2013; 7:666. doi:10.3389/fnhum.2013.00666.

28. Hamilton JP, Farmer M, Fogelman P, Gotlib IH. Depressive rumination, the default-mode network, and the dark matter of clinical neuroscience. Biol Psychiatry. 2015; 78(4): 224-230. doi:10.1016/j.biopsych.2015.02.020.

29. Johansen-Berg H, Gutman DA, Behrens TEJ, et al. Anatomical connectivity of the subgenual cingulate region targeted with deep brain stimulation for treatment-resistant depression. Cereb Cortex. 2008; 18(6): 1374-1383. doi:10.1093/cercor/bhm167.

30. Dunlop BW, Rajendra JK, Craighead WE, et al. Functional connectivity of the subcallosal cingulate cortex and differential outcomes to treatment with cognitive-behavioral therapy or antidepressant medication for major depressive disorder. Am J Psychiatry. 2017; 174(6): 533-545. doi:10.1176/appi. ajp.2016.16050518.

31. Williams KD, Jarvis B. Cyberball: a program for use in research on interpersonal ostracism and acceptance. Behav Res Methods. 2006 38(1): 174-180.

32. Eisenberger NI, Lieberman MD, Williams KD. Does rejection hurt? An FMRI study of social exclusion. Science. 2003; 302(5643): 290-292. doi:10.1126/science.1089134.

33. Rotge JY, Lemogne C, Hinfray S, et al. A meta-analysis of the anterior cingulate contribution to social pain. Soc Cogn Affect Neurosci. 2015; 10(1): 19-27. doi:10.1093/scan/nsu110.

34. Hughes BL, Beer JS. Protecting the self: the effect of socialevaluative threat on neural representations of self. J Cogn Neurosci. $2013 ; \mathbf{2 5}(4)$ : 613-622. doi:10.1162/jocn_a_00343. 
35. Gao S, Assink M, Cipriani A, Lin K. Associations between rejection sensitivity and mental health outcomes: a meta-analytic review. Clin Psychol Rev. 2017; 57: 59-74. doi:10.1016/j.cpr.2017.08.007.

36. Pearson KA, Watkins ER, Mullan EG. Rejection sensitivity prospectively predicts increased rumination. Behav Res Ther. 2011; 49(10): 597-605. doi:10.1016/j.brat.2011.06.004.

37. Kendler KS, Hettema JM, Butera F, Gardner CO, Prescott CA. Life event dimensions of loss, humiliation, entrapment, and danger in the prediction of onsets of major depression and generalized anxiety.
Arch Gen Psychiatry. 2003; 60(8): 789-796. doi:10.1001/ archpsyc.60.8.789.

38. Ayduk O, Downey G, Kim M. Rejection sensitivity and depressive symptoms in women. Pers Soc Psychol Bull. 2001; 27 (7): 868-877.

39. Clemens B, Wagels L, Bauchmüller M, Bergs R, Habel U, Kohn N. Alerted default mode: functional connectivity changes in the aftermath of social stress. Sci Rep. 2017; 7: 40180. doi:10.1038/ srep40180. 